\title{
The Adomian Decomposition Method for a Type of Fractional Differential Equations
}

\author{
Peng Guo \\ Business School, Shanghai Dianji University, Shanghai, China \\ Email: guop@sdju.edu.cn
}

How to cite this paper: Guo, P. (2019) The Adomian Decomposition Method for a Type of Fractional Differential Equations. Journal of Applied Mathematics and Physics, 7, 2459-2466.

https://doi.org/10.4236/jamp.2019.710166

Received: September 23, 2019

Accepted: October 21, 2019

Published: October 24, 2019

Copyright (อ 2019 by author(s) and Scientific Research Publishing Inc. This work is licensed under the Creative Commons Attribution International License (CC BY 4.0).

http://creativecommons.org/licenses/by/4.0/

\begin{abstract}
Fractional differential equations are widely used in many fields. In this paper, we discussed the fractional differential equation and the applications of Adomian decomposition method. Where the fractional operator is in Caputo sense. Through the numerical test, we can find that the Adomian decomposition method is a powerful tool for solving linear and nonlinear fractional differential equations. The numerical results also show the efficiency of this method.
\end{abstract}

\section{Keywords}

Caputo, Fractional Integral, Adomian Decomposition Method, Approximate

\section{Introduction}

Fractional calculus can be dated back to the end of $17^{\text {th }}$ century. In 1695, Leibniz and L'Hospital have discussed 1/2 order derivative, which is regarded as the birth of fractional differential equation. For a long time, fractional calculus does not attract enough attention. It is only considered and studied by many mathematicians. However, in last few decades, fractional calculus has been studied more and more in applied sciences and engineering. The fractional derivative has been applied in many physical problems such as frequency-dependent damping behavior of materials, motion of a large thin plate in a Newtonian fluid, creep and relaxation functions for viscoelastic materials etc. There are many authors who have demonstrated the application of fractional derivative like Oldham and Spainer [1], Miller and Ross [2], Podlubny [3], Samko et al. [4], Hilfer [5]. And more recently Sabiter et al. [6] have demonstrated the development and application of fractional calculus in physical and engineering. Other applications of fractional differential equations we can refer to [7]-[12]. 
Fractional calculus is found to be more suitable modeling the process with long range interaction and physical problems described by fractional equations, but sometimes it's difficult to get the solution of fractional differential equations. For that reason, we need a reliable and efficient technique for solving fractional differential equations. In [13], Tamsir and Srivastava give an analytical study for time fractional Klein-Gordon equation. Chen et al. use the discrete method to study the time fractional Klein-Gordon equation [14]. Fewer researchers consider giving an approximate solution. In this paper, we give an analytical solution of the time fractional differential equation of the following form

$$
{ }_{C} D_{0, t}^{\alpha} u=\frac{\partial^{2} u}{\partial x^{2}}+F(u) \text {. }
$$

where, ${ }_{C} D_{0, t}^{\alpha}$ is the fractional operator in Caputo sense, $1<\alpha \leq 2$. Except Caputo fractional derivative, there are many other different fractional derivatives, such as the Riemann-Liouville fractional derivative, Grünwald-Letnikov fractional derivative, Rietz fractional derivative etc. From the pure mathematical point, Riemann-Liouville derivative is somewhat more popular than Caputo derivative. Many earlier researchers use it instead of Caputo derivative, but for the Riemann-Liouville derivative we need to specify the values of certain fractional derivatives of the unknown solution at the initial conditions. However, when we deal with the concrete physical problem, the fractional derivative doesn't have physical meaning. When we deal with the Caputo derivatives, we may only specify the integer order derivative. It has a clearly physical meaning and can be measured. Another reason we choose Caputo derivative is that under homogeneous conditions the equations with Riemann-Liouville operator are equivalent to the equations with Caputo operator, if we choose Caputo derivative it allows us to specify inhomogeneous initial conditions, too, if we needed.

This paper is organized as follows. In Section 2, we discuss some basic properties about fractional derivative and fractional integral which will be used in the following part. In Section 3, we introduce the Adomian decomposition method, and the detailed Scheme about the time fractional differential Equation (1.1) will be discussed. In Section 3, a numerical test will be showed, the approximate solution will be compared with the exact solution, and the error analysis will be given.

\section{Fractional Integral and Fractional Derivative}

First, we will give some definitions about fractional calculus including fractional integral and fractional derivative. For fractional derivative there are already exist several different definitions and in general these different definitions are not equivalent to each other. Here we only give the most common definition.

Definition 2.1. If $f(x)$ is continuous on $(0,+\infty)$ and $\alpha>0$ then the fractional integral is defined as

$$
D_{0, t}^{-\alpha} f(t)=\frac{1}{\Gamma(\alpha)} \int_{0}^{t}(t-\tau)^{\alpha-1} f(\tau) \mathrm{d} \tau .
$$


Definition 2.2. If $f^{(n)}(x)$ is continuous on $(0,+\infty)$ and $n-1<\alpha \leq n$ then the Caputo fractional derivative is defined as

$$
{ }_{C} D_{0, t}^{-\alpha} f(t)=\frac{1}{\Gamma(n-\alpha)} \int_{0}^{t}(t-\tau)^{n-\alpha-1} f^{(n)}(\tau) \mathrm{d} \tau .
$$

Property 2.1. [3] If $f(x)$ is continuous on $(0,+\infty)$ and $\alpha>0, \beta>0$ then

$$
D_{0, t}^{-\alpha} D_{0, t}^{-\beta} f(t)=D_{0, t}^{-\alpha-\beta} f(t)
$$

Property 2.2. [3] If $f^{(n)}(x)$ is continuous on $(0,+\infty)$ and $n-1<\alpha \leq n$ then

$$
{ }_{C} D_{0, t}^{\alpha} D_{0, t}^{-\alpha} f(t)=f(t)
$$

Property 2.3. [3] If $f^{(n)}(x)$ is continuous on $(0,+\infty)$ and $n-1<\alpha \leq n$ then

$$
D_{0, t \quad C}^{-\alpha} D_{0, t}^{\alpha} f(t)=f(t)-\sum_{k=0}^{n-1} \frac{f^{(k)}(0)}{k !} t^{k} .
$$

Here, we only give some basic properties about Caputo fractional derivative and fractional integral which we will use in the following part. For some other properties about Caputo fractional derivative and other definitions about fractional calculus we can refer to [4].

\section{Adomian Decomposition Method}

The Adomian decomposition method [15] [16] is powerful tool for solving linear or nonlinear equations. For every nonlinear differential equation can be decomposed into the following form

$$
L(u)+R(u)+N(u)=g,
$$

where $L$ is the highest order differential operator, $R u$ is the remainder of the linear part, $\mathrm{Nu}$ represents the nonlinear part and $g$ is a given function. In general, the operator $L$ is invertible. If we take $L^{-1}$ on both sides of Equation (3.1), an equivalent expression can be given,

$$
u=-L^{-1} R(u)-L^{-1} N(u)+L^{-1} g+\varphi,
$$

where $\varphi$ satisfy $L \varphi=0$ and the initial conditions. If $L$ is the second order derivative, $L^{-1}$ is the two-fold definite integral. For the Adomian decomposition method, the solution $u$ is expressed in terms of a series form,

$$
u=\sum_{n=0}^{\infty} u_{n}
$$

The nonlinear term $N(u)$ is represented by the Adomian polynomials $A_{n}$, i.e.

$$
N(u)=\sum_{n=0}^{\infty} A_{n} .
$$

$A_{n}$ depends on $u_{0}, u_{1}, \cdots, u_{n}$ and can be formulated by 


$$
A_{n}=\frac{1}{n !} \frac{\mathrm{d}^{n}}{\mathrm{~d} \lambda^{n}}\left[N\left(\sum_{k=0}^{\infty} \lambda^{k} u_{k}\right)\right]_{\lambda=0}, n=0,1,2, \cdots
$$

For clarity, first few several items of the Adomian polynomials will be listed

$$
\left\{\begin{aligned}
A_{0} & =N\left(u_{0}\right) \\
A_{1} & =u_{1} N^{(1)}\left(u_{0}\right) \\
A_{2} & =u_{2} N^{(1)}\left(u_{0}\right)+\frac{1}{2 !} u_{1}^{2} N^{(2)}\left(u_{0}\right) \\
A_{3}= & u_{3} N^{(1)}\left(u_{0}\right)+u_{1} u_{2} N^{(2)}\left(u_{0}\right)+\frac{1}{3 !} u_{1}^{3} N^{(3)}\left(u_{0}\right) \\
A_{4}= & u_{4} N^{(1)}\left(u_{0}\right)+\left[\frac{1}{2 !} u_{2}^{2}+u_{1} u_{3}\right] N^{(2)}\left(u_{0}\right)+\frac{1}{2 !} u_{1}^{2} u_{2} N^{(3)}\left(u_{0}\right)+\frac{1}{4 !} u_{1}^{4} N^{(4)}\left(u_{0}\right), \\
& \vdots
\end{aligned}\right.
$$

Then for the Equation (3.1), we have

$$
\sum_{n=0}^{\infty} u_{n}=-L^{-1} R \sum_{n=0}^{\infty} u_{n}-L^{-1} \sum_{n=0}^{\infty} A_{n}+L^{-1} g+\varphi .
$$

The Adomian's technique is equivalent to the following relation which can be defined as

$$
\left\{\begin{aligned}
u_{0} & =L^{-1} g+\varphi, \\
u_{1} & =-L^{-1} R\left(u_{0}\right)-L^{-1} A_{0}, \\
u_{2} & =-L^{-1} R\left(u_{1}\right)-L^{-1} A_{1}, \\
& \vdots \\
u_{n} & =-L^{-1} R\left(u_{n-1}\right)-L^{-1} A_{n-1}, \\
& \vdots
\end{aligned}\right.
$$

In theory, if we calculate all the terms $u_{n}$ we can find the exact solution. In fact, we just need to compute the first finite terms. Cherruault et al. have proved the convergence of Adomian decomposition method [17] [18]. From some numerical tests of the following part, we can find that the sum of the first three or four terms has high accuracy. The more terms we calculate, the higher the accuracy.

\section{Numerical Examples}

In order to verify the accuracy of the method which described in the last section, two numerical examples will be considered.

Example 1. ${ }_{C} D_{0, t}^{\alpha} u=\frac{\partial^{2} u}{\partial x^{2}}+u$, subject to the initial conditions $u(x, 0)=1+\sin x$, $u_{t}(x, 0)=0$.

First, we take $D_{0, t}^{-\alpha}$ on both sides of the example 1 , the following relation is given

$$
u=u(x, 0)+t u_{t}(x, 0)+\frac{1}{\Gamma(\alpha)} \int_{0}^{t}(t-\tau)^{\alpha-1}\left(u_{x x}(x, \tau)+u(x, \tau)\right) \mathrm{d} \tau
$$

With the scheme we discussed in the last part, we have 


$$
\left\{\begin{array}{c}
u_{0}=1+\sin x, \\
u_{1}=\frac{t^{\alpha}}{\Gamma(\alpha+1)}, \\
u_{2}=\frac{t^{2 \alpha}}{\Gamma(2 \alpha+1)}, \\
\vdots
\end{array}\right.
$$

Then the approximate solution is

$\hat{u}=1+\sin x+\frac{t^{\alpha}}{\Gamma(\alpha+1)}+\frac{t^{2 \alpha}}{\Gamma(2 \alpha+1)}+\cdots+\frac{t^{n \alpha}}{\Gamma(n \alpha+1)}$. In order to test the accuracy of the approximate solution, we consider when $\alpha=2$, the exact solution is $u(x, t)=\sin x+\cosh t$. Figure 1 shows the exact solution and the approximate solution with the first four terms.

Table 1 shows the error of the exact solution and the approximate solution. In this example, we only use the forst four terms to approximate the exact solution. From the error column we can find that the absolute error is very small, the Adomian decomposition method has a high convergence order. The more terms we use, the higher accuracy we get.

Example 2. ${ }_{C} D_{0, t}^{\alpha} u=\frac{\partial^{2} u}{\partial x^{2}}-\sin u, 1<\alpha \leq 2$ subject to the initial conditions $u(x, 0)=\sin \pi x, u_{t}(x, 0)=0$.

Similarly, with the procedure we used in the first example, we have the following result about $u_{i}$

$$
\left\{\begin{aligned}
u_{0}= & \sin \pi x, \\
u_{1}= & \frac{t^{\alpha}\left(-\pi^{2} \sin (\pi x)-\sin (\sin (\pi x))\right)}{\Gamma(\alpha+1)}, \\
u_{2}= & \frac{t^{2 \alpha}}{\Gamma(2 \alpha+1)}\left(\pi^{4} \sin (\pi x)+\pi^{2} \sin (\sin (\pi x)) \cos ^{2}(\pi x)\right. \\
& +2 \pi^{2} \sin (\pi x) \cos (\sin (\pi x))+\cos (\sin (\pi x)) \sin (\sin (\pi x)), \\
& \vdots
\end{aligned}\right.
$$

In this example we use the sum of the first three terms as the approximate solution of the problem we discussed. When we consider $\alpha=2$, the exact solution is $u(x, t)=\frac{1}{2}(\sin (\pi(x+t))+\sin (\pi(x-t)))$. Figure 2 shows the exact solution and the approximate solution.

Table 2 shows the exact solution and approximate solution of the nonlinear fractional differential equation. In the last column we can find that the absolute error is small, here, we only use the first three terms to approximate the solution. If we use more terms, the approximation works better.

\section{Conclusion}

In this work, the Adomian decomposition method is applied to solving a time fractional differential equation. Both the linear and nonlinear type of fractional 

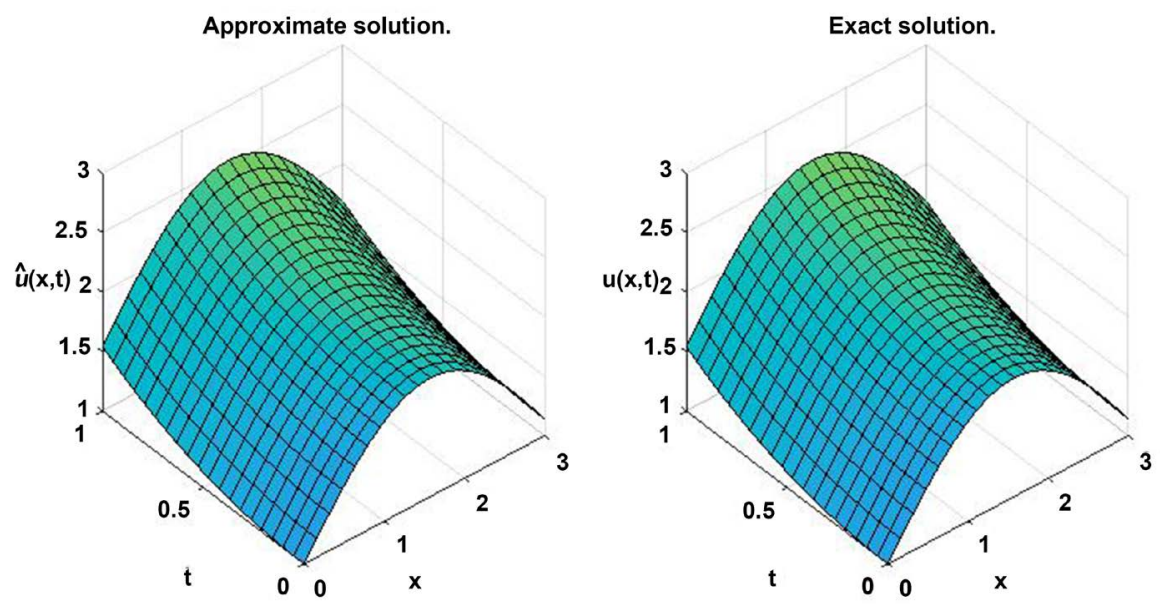

Figure 1. Approximate solution and exact solution of example 1.
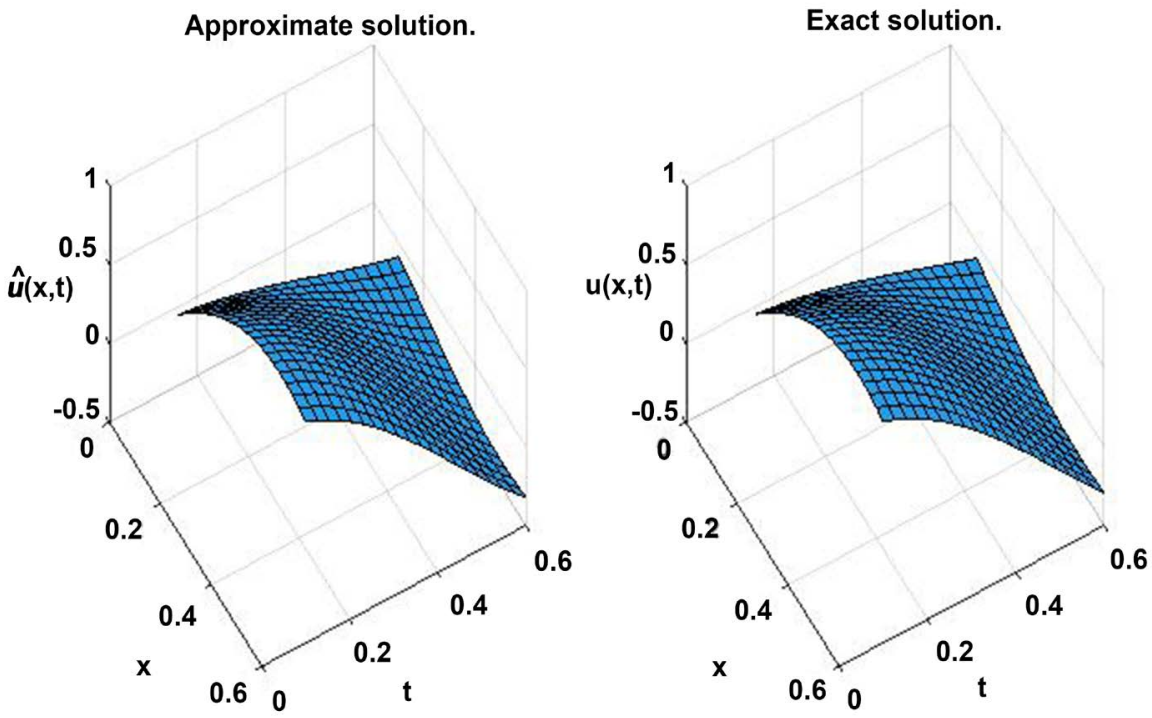

Figure 2. Approximate solution and exact solution of example 2.

Table 1. Error of exact solution and approximate solution, where $x=1$.

\begin{tabular}{cccc}
\hline $\mathrm{t}$ & Exact solution & $\mathrm{ADM}$ & Error \\
\hline 0.10 & 1.846475152863700 & 1.846475152863700 & $0.0 \mathrm{E}-16$ \\
0.15 & 1.852742094384567 & 1.852742094384565 & $2.0 \mathrm{E}-15$ \\
0.20 & 1.861537740426972 & 1.861537740426944 & $2.8 \mathrm{E}-14$ \\
0.25 & 1.872884084687470 & 1.872884084687207 & $2.6 \mathrm{E}-13$ \\
0.30 & 1.886809498936757 & 1.886809498935129 & $1.6 \mathrm{E}-12$ \\
0.35 & 1.903348803963882 & 1.903348803956273 & $7.6 \mathrm{E}-12$ \\
0.40 & 1.922543356646351 & 1.922543356617420 & $2.8 \mathrm{E}-11$ \\
0.45 & 1.944441153363867 & 1.944441153269889 & $9.3 \mathrm{E}-11$ \\
0.50 & 1.969096950014277 & 1.969096949744652 & $2.7 \mathrm{E}-10$ \\
\hline
\end{tabular}


Table 2. Error of exact solution and approximate solution, where $x=1$.

\begin{tabular}{cccc}
\hline $\mathrm{t}$ & Exact solution & ADM & Error \\
\hline 0.010 & 0.999506560365732 & 0.999464491451072 & $4.2 \mathrm{E}-5$ \\
0.015 & 0.998889874961970 & 0.998795232947192 & $9.5 \mathrm{E}-5$ \\
0.020 & 0.998026728428272 & 0.997858508448686 & $1.6 \mathrm{E}-4$ \\
0.025 & 0.996917333733128 & 0.996654555362476 & $2.6 \mathrm{E}-4$ \\
0.030 & 0.995561964603080 & 0.995183678926039 & $3.7 \mathrm{E}-4$ \\
0.035 & 0.993960955455180 & 0.993446252207397 & $5.1 \mathrm{E}-4$ \\
0.040 & 0.992114701314478 & 0.991442716105125 & $6.7 \mathrm{E}-4$ \\
0.045 & 0.990023657716558 & 0.989173579348348 & $8.5 \mathrm{E}-4$ \\
0.050 & 0.987688340595138 & 0.986639418496738 & $1.1 \mathrm{E}-3$ \\
\hline
\end{tabular}

differential equations are considered. From the numerical result, we can find that the Adomian decomposition method is an efficient algorithm. We use only first several terms to approximate the exact solution, the numerical result has high precision. In general, some differential equations are hard to deal with because of the nonlinear terms. The Adomian decomposition method is a powerful tool to cope with this problem. Moreover, no linearization or perturbation is required in this method.

\section{Acknowledgements}

This research was funded by the Humanity and Social Science Youth Foundation of Ministry of Education (No. 18YJC630120), the Applied Mathematics of Shanghai Dianji University (No. 16JCXK02).

\section{Conflicts of Interest}

The author declares no conflicts of interest regarding the publication of this paper.

\section{References}

[1] Oldham, K.B. and Spainer, J. (1974) The Fractional Calculus. Academic Press, New York.

[2] Miller, K.S. and Ross, B. (1993) An Introduction to the Fractional Calculus and Fractional Differential Equations. John Wily and Sons Inc., New York.

[3] Podlubny, I. (1999) Fractional Differential Equations. Academic Press, New York.

[4] Samko, S.G., Kilbas, A.A. and Marichev, O.I. (1993) Fractional Integrals and Derivatives: Theory and Applications. Gordon and Breach Science Publishers, Yverdon.

[5] Hilfer, R. (2000) Applications of Fractional Calculus in Physics. World Scientific, Singapore. https://doi.org/10.1142/9789812817747

[6] Sabatier, J., Argawal, O.P. and Machado, A.T. (2007) Advances in Fractional Calculus. Springer, Dordrecht. https://doi.org/10.1007/978-1-4020-6042-7

[7] Bagley, R.L. and Torvik, J. (1983) Fractional Calculus-A Different Approach to the 
Analysis of Viscoelastically Damped Structures, AIAA Journal, 21, 741-748. https://doi.org/10.2514/3.8142

[8] Momani, S. and Al-Khaled, K. (2005) Numerical Solutions for Systems of Fractional Differential Equations by the Decomposition Method. Applied Mathematics and Computation, 162, 1351-1365. https://doi.org/10.1016/j.amc.2004.03.014

[9] Momani, S. (2006) Non-Perturbative Analytical Solutions of the Space- and Time-Fractional Burgers Equations. Chaos, Solitons and Fractals, 28, 930-937. https://doi.org/10.1016/j.chaos.2005.09.002

[10] Chen, Y. and Moore, K. (2002) Discretization Schemes for Fractional-Order Differentiators and Integrators. IEEE Transactions on Circuits and Systems I: Fundamental Theory and Applications, 49, 363-367. https://doi.org/10.1109/81.989172

[11] Song, B., Xu, L. and Lu, X. (2015) Discrete Approximation of Fractional-Order Differentiator Based on Tustin Transform. Science Technology and Engineering, 15, 92-95.

[12] Varieschi, G. (2018) Applications of Fractional Calculus to Newtonian Mechanics. Journal of Applied Mathematics and Physics, 6, 1247-1257.

https://doi.org/10.4236/jamp.2018.66105

[13] Tamsir, M. and Srivastava, V.K. (2016) Analytical Study of Time-Fractional Order Klein-Gordon Equation. Alexandria Engineering Journal, 55, 561-567. https://doi.org/10.1016/j.aej.2016.01.025

[14] Chen, H., Lü, S. and Chen, W. (2017) A Fully Discrete Spectral Method for the Nonlinear Time Fractional Klein-Gordon Equation. Taiwanese Journal of Mathematics, 21, 231-251. https://doi.org/10.11650/tjm.21.2017.7357

[15] Adomian, G. (1988) A Review of the Decomposition Method in Applied Mathematics. Journal of Mathematical Analysis \& Applications, 135, 501-544. https://doi.org/10.1016/0022-247X(88)90170-9

[16] Adomian, G. (1994) Solution of Nonlinear Evolution Equations. Mathematical and Computer Modelling, 20, 1-2. https://doi.org/10.1016/0895-7177(94)90120-1

[17] Cherruault, Y., Adomian, G., Abbaoui, K. and Rach, R. (1995) Further Remarks on Convergence of Decomposition Method. International Journal of Bio-Medical Computing, 38, 89-93. https://doi.org/10.1016/0020-7101(94)01042-Y

[18] Abbaoui, K. and Cherruault, Y. (1995) New Ideas for Proving Convergence of Decomposition Methods. Computers \& Mathematics with Applications, 29, 103-108. https://doi.org/10.1016/0898-1221(95)00022-Q 